formation of soluble sugars. Internal transformations involving no losses of dry-matter may also have occurred. Variation in the contents of total nonvolatile ether-soluble acids and malic acid was appreciable, but for oxalic and citric acids it was relatively small. Nicotine content did not vary during curing. No certain losses of total nitrogen were found. The $p \mathrm{H}$ value of the leaf did not vary. Neither soluble phosphate, soluble sulphur and protein-sulphur contents, nor soluble calcium content, varied during curing of the leaf.

\section{Curing of Sheepskins in the Sudan}

A LARGE-SCALE experiment on the curing of sheepskins has been carried out in the Sudan. The straight drying of sheepskins was compared with dry salting in the hot and in the cold seasons; drying in the sun was compared with shade-drying, and ropedrying with framing. At each season nine batches of fifty skins each were treated, the skins later being dressed in England as white suede. Straight drying gave almost consistently the best leather and cold season 'cure' was better than hot. Damage was found to be present in the raw skins although it could only be recognized microscopically. In its milder form it caused the grain to be rubbed off during processing or caused holes to appear in the skins ; extreme damage led to complete disintegration and the skins dropped to pieces. It is suggested that an immediate improvement in the quality of 'curing' in the Sudan might be achieved by abandoning dry-salting, and by straight-drying the skins without delay, avoiding excessive heat in the direct sun (Colonial Plant and Animal Products, 3, No. 4: 1952-53).

\section{Formation of Asparagine in Lupin Seedlings}

THE formation of asparagine in etiolated seedlings of Lupinus albus has been considered in some detail on the basis of analytical and experimental investigations by A. N. Meiss (Bull. Connecticut $A$ ric. Exp. Sta., No. 553, $1 ;$ 1952). In this bulletin recent work on the biochemistry of asparagine formation is reviewed, while in the experimental section the germination and growth of etiolated seedlings are described and comprehensive analyses of these materials submitted, reference being made to the nitrogen fractions, lipids, soluble and insoluble carbohydrates, organic acids, moisture and ash. In the discussion of the experimental data, the relation of asparagine accumulation and growth, the sources of the asparagine nitrogen and carbohydrate, and the role of the organic acids and mineral nutrients are considered. The principal conclusion which is drawn is that the large accumulation of asparagine in etiolated seedlings of Lupinus is a consequence of a normal, but obligatory, utilization of seed protein as a respiratory substrate. The asparagine which arises by secondary synthesis-in the Lupinus seedlings it is about 90 per cent of the total formed-is considered to be a product of protein respiration, and as such is not metabolized further until acute starvation conditions prevail. Other observations and results which are associated with this conclusion are set out and discussed in detail.

\section{Growth of Excised Roots}

Is one of a series of studies of the growth of excised roots in controlled aseptic culture, J. C. Skinner and H. E. Street have investigated the cultural requirements of a number of strains of
Senecio vulgaris (groundsel), which also have been, and are being, studied genetically (New Phyt., 53, I, 44 ; 1954). Earlier work, in which it was found that excised roots of strains within a species may show clearly defined differences in their capacity for 'essential metabolite' synthesis, is reviewed. The underlying point of view in these new physiologicalgenetical studies is that more detailed examination of the differences in the growth requirements of different strains should enable a comparative physiology of excised root systems to be constructed. The differences observed in the growth responses of cultured excised roots of tomato strains to added B vitamins suggests that this technique may be used to advantage in the study of the genetical control of synthetic processes in flowering plants. Seeds of a number of geographical strains of Senecio vulgaris were available and excised roots have now been successfully cultured. The present paper gives details of a technique for the establishment and maintenance of excised root clones and describes the growth responses of one clone to a range of nutritive treatments. Subsequent work has involved comparison of the growth in selected culture media of the roots of a number of geographical strains and of hybrids between them, and study of the segregation of excised root characters in the $F_{2}$ generations of these crosses.

\section{Flake-Tool Cultures in South Africa}

$A_{N}$ article by Prof. T. F. Dreyer, entitled "The Origin and Chronology of the Fauresmith Culture" (Res. Nat. Museum, Bloemfontein, 1, Part 3 ; 1953), makes an interesting suggestion as to the origin of the flake-tool cultures which appear with some suddenness at the end of Fauresmith times. The current view of many prehistorians in South Africa is that the Middle Stone Age complex (flake-tool industries) is directly derived from the Stellenbosch (core-tool industries), the Fauresmith being the latest of the Stellenbosch series. It is not easy to explain why this change should have taken place at the time it did and why the coups de poing which had persisted for perhaps scores of thousands of years throughout many changes of climate and conditions should have so rapidly disappeared and been replaced by others so completely different, unless influences from outside had operated. In Europe and northern Asia, where a similar phenomenon occurs, it can be shown from distribution maps that the flake-tool making cultures existed farther to the east contemporarily with the core-tool making people, and replaced them in western Europe towards the end of Würm I times. Prof. Dreyer suggests a somewhat similar solution for the problem in South Africa in that he believes the Florisbad (flake-tool) culture to be older in time than has been hitherto allowed, and that it was the influence of this culture on the Fauresmith that led to the development of the Middle Stone Age; and that it was fertilization by the Florisbad culture that gave rise to the profound changes which took place towards the end of the Fauresmith period. Certainly the natures of the two cultures were entirely different. The coup de poing folk were conservative and uniform in their implement evolution - the development of the industries throughout millennia is the same whether in South Africa, East Africa or Western Europe ( = the Chelleo-Acheulean culture). The flake-tool makers were quite different, always experimenting and producing changes and new types of tools. It is difficult to see how the one 